\title{
Nasal Drops, Lyophilisate for Suspension Dosage Form
}

National Cancer Institute

\section{Source}

National Cancer Institute. Nasal Drops, Lyophilisate for Suspension Dosage Form. NCI

Thesaurus. Code C149678.

Solid preparation for veterinary use consisting of a freeze-dried powder intended to be dispersed in the specified liquid to obtain a suspension for use as nasal drops. 